\title{
El impacto de los pies para la salud
}

\author{
The impact of the foot for the health
}

\begin{abstract}
Los pies son una parte esencial en el mantenimiento y en el control de la salud de las personas. Las especiales características óseas, musculares, ligamentosas, nerviosas, vasculares, variantes anatómicas ${ }^{1}$ y los cambios producidos a lo largo de la vida hacen necesario la supervisión, la evaluación y el cuidado de los mismos para contribuir a la mejora de la calidad de vida, el bienestar, la independencia y la autonomía de las personas ${ }^{2}$.
\end{abstract}

Por ello, se hace patente la necesidad en determinar el impacto del pie para la salud y convertirse en la prioridad número 1 para el Sistema Nacional de Salud (SNS) y el Ministerio de Sanidad, Consumo y Bienestar Social a nivel nacional, que deben incorporar la Podología dentro de la cartera de prestaciones sanitarias al tratarse la salud del pie de un factor predictor en la mejora de las enfermedades crónicas como la hipertensión arterial, la hipercolesterolemia, la obesidad, el estrés, la diabetes, las cardiopatías, la artritis reumatoidea, la dermatitis atópica, la ansiedad, la depresión, la osteoporosis, entre otras, cuando se establecen planes de cuidados vinculados a la deambulación, demostrando una mejora significativa en el control de las enfermedades no transmisibles ${ }^{3}$.

Además, las variaciones que se producen vinculadas a la edad, los estilos de vida inadecuados, los factores ambientales y la biología humana facilitan la aparición de deformidades y alteraciones en los pies con un incremento establecido que oscila entre el 71 y el $87 \%$, lo cual genera un motivo frecuente de atención médica, podológica y del personal de enfermería $^{4,5}$, que repercuten en la pérdida de estabilidad postural, limitación funcional, dolor, dificultad para calzarse e incremento de caídas, las cuales suponen un aumento del gasto sanitario y una demanda por parte de las instituciones y de la sociedad de una mejora de la atención de la salud de los pies.
Por lo tanto, el actual paradigma a nivel de la salud tiene que contemplar la incorporación de podólogos para contribuir a optimizar los recursos, la atención y el gasto sanitario donde el impacto del pie para la salud, unido a la prestación podológica, pueden contribuir a disminuir hasta en un 80 \% las amputaciones del pie diabético y una reducción significativa de los recursos y de los costes económicos de la diabetes ${ }^{6}$. En lo que atañe al autocuidado del pie, favorece una mejora de la limitación funcional y reduce los riesgos de la aparición de una discapacidad en el pie o en el miembro inferior, con especial interés en las personas mayores ${ }^{7}$. Del mismo modo, en el caso de las consultas podológicas, reduce la necesidad de consultas quirúrgicas, el dolor, riesgo de caídas ${ }^{8}$ y derivaciones a atención especializada ante la sospecha patológica o en estadios iniciales para detectar y aplicar tratamientos que impidan, retrasen o corrijan el desarrollo de la enfermedad.

Por ello, el impacto actual hace necesario que se establezcan planes de cuidados que repercutan en la promoción activa de la salud del pie y que se establezcan sinergias con la Podología en la búsqueda de establecer estrategias orientadas a la prevención de la salud, que permitan la disminución de la prevalencia de afecciones y deformidades de los pies, que a su vez reduzcan la carga de morbilidad atribuida a las enfermedades degenerativas, que se prevé que para el año 2030 serán responsables de las tres cuartas partes del total de las defunciones ${ }^{9}$.

Basada en estos antecedentes, es evidente la necesidad de la atención de la salud del pie en la busca de prevenir la aparición de alteraciones y deformidades en los pies, teniendo como objetivo empoderar, controlar y mejorar la salud de las personas. 
Por ello, se constata la transversalidad que tiene la salud del pie y su repercusión para el control de la salud, la autonomía, la calidad de vida y el bienestar de las personas, que hace necesaria la incorporación de la Podología como nexo que permita incrementar y mejorar la toma de decisiones profesionales en la práctica de la salud del pie en el SNS, como consecuencia de las listas de espera a las que se enfrentan los facultativos, la priorización por un manejo específico que requieren los pacientes a la hora de recibir un tratamiento a nivel del pie y la poca relevancia dada a la salud del pie, la cual propicia un impacto financiero sobre el sistema de salud, gran morbilidad y mortalidad prematura, alto grado de discapacidad, dependencia, y un deterioro significativo de la calidad de vida de los afectados, siendo el SNS un elemento clave en mejorar las condiciones del pie de manera especial, y de la salud de forma general.

El contexto actual debe impulsar y ofrecer oportunidades para el avance del futuro de la salud pública donde el pie, el podólogo, pueden contribuir a reducir el impacto de la morbilidad y mortalidad y dotar de políticas que establezcan herramientas y espacios saludables que sean equitativas para todas las personas.

Daniel López López Research, Health and Podiatry Group. Department of Health Sciences. Faculty of Nursing and Podiatry. Universidade da Coruña, Spain

\section{BIBLIOGRAFÍA}

1. Munuera Martínez PV. El primer radio. Biomecánica y ortopodología. Santander: EXA; 2012

2. Ramos-Galván J, Álvarez-Ruiz V, Tovaruela-Carrión N, Mahillo-Durán R, Gago-Reyes F. Population impact of a podiatric school health programme. Gac Sanit. 2016;30(2):137-9. DOI: 10.1016/j.gaceta.2015.12.002.

3. Williams PT, Thompson PD. Walking versus running for hypertension, cholesterol, and diabetes mellitus risk reduction. Arterioscler Thromb Vasc Biol. 2013;33(5):1085-1. DOI: 10.1161/ATVBAHA.112.300878.

4. Dawson J, Thorogood M, Marks SA, Juszczak E, Dodd C, Lavis G, et al. The prevalence of foot problems in older women: A cause for concern. J Public Health Med. 2002;24(2):77-84. DOI: 10.1093/pubmed/24.2.77.

5. Pita-Fernandez S, Gonzalez-Martin C, Seoane-Pillado T, Pertega-Diaz S, Perez-Garcia S, Lopez-Calvino B. Podiatric medical abnormalities in a random population sample 40 years or older in Spain. J Am Pod Med Assoc. 2014;104(6):574-82. DOI: 10.7547/8750-7315-104.6.574.

6. Krishnan S, Nash F, Baker N, Fowler D, Rayman G. Reduction in diabetic amputations over 11 years in a defined UK. population: benefits of multidisciplinary team work and continuous prospective audit. Diabetes Care. 2008;31(1):99-101. DOI: 10.2337/dc07-1178.

7. Waxman R, Woodburn H, Powell M, Woodburn J, Blackburn S, Helliwell P. FOOTSTEP: a randomized controlled trial investigating the clinical and cost effectiveness of a patient self-management program for basic foot care in the elderly. J Clin Epidemiol. 2003;56(11):1092-9. DOI: 10.1016/s0895-4356(03)00197-5.

8. Bonanno DR, Medica VG, Tan DS, Spring AA, Bird AR, GazarekJ. Evaluating the outcomes of a podiatry-led assessment service in a public hospital orthopaedic unit. J Foot Ankle Res. 2014;7(1):45. DOI: 10.1186/ s13047-014-0045-6.

9. WHO | Global status report on noncommunicable diseases 2014. WHO. 2015. 